\title{
Electrostatic Contributions to Chain Stiffness and Excluded-Volume Effects in Sodium Poly(2-acrylamido-2-methylpropanesulfonate) Solutions
}

\author{
Ryo Hagino, Junko YASHIRo, Michiko SAKATA, and Takashi NORISUYE ${ }^{\dagger}$ \\ Department of Macromolecular Science, Graduate School of Science, Osaka University, \\ 1-1 Machikaneyama-cho, Toyonaka, Osaka 560-0043, Japan
}

(Received April 17, 2006; Accepted May 9, 2006; Published July 24, 2006)

\begin{abstract}
Chain stiffness and excluded-volume effects in $0.005-1 \mathrm{M}$ aqueous $\mathrm{NaCl}$ solutions of sodium poly(2acrylamido-2-methylpropanesulfonate) (Na PAMPS) at $25^{\circ} \mathrm{C}$ have been studied by viscometry on 15 samples with weight-average molecular weights of $1.7 \times 10^{3}-7.1 \times 10^{5}$. The molecular weight dependence of intrinsic viscosity at each $\mathrm{NaCl}$ concentration $C_{\mathrm{s}}$ is analyzed by a combination of Yoshizaki et al.'s theory for unperturbed wormlike chains and the quasi-two-parameter theory for excluded-volume effects to estimate the persistence length $q$ and the excluded-volume strength $B$ as functions of $C_{\mathrm{s}}$. Both $q$ and its electrostatic contribution $q_{\mathrm{el}}$ are close to the previously estimated values for $\mathrm{Na}$ poly(styrenesulfonate) (Na PSS) at the same $C_{\mathrm{s}}$, while $B$ considerably differs between the two polymers. As was found for Na PSS, available polyelectrolyte theories fail to describe the $C_{\mathrm{s}}$-dependence of $q_{\mathrm{el}}$ and $B$ for Na PAMPS. [doi:10.1295/polymj.PJ2006013]

KEY WORDS Polyelectrolyte / Poly(2-acrylamido-2-methylpropanesulfonate) / Wormlike Chain / Chain Stiffness / Electrostatic Persistence Length / Excluded-Volume Effect /
\end{abstract}

The persistence length $q$ of a polyelectrolyte, modeled by the wormlike chain, ${ }^{1}$ in aqueous salt increases with lowering salt concentration $C_{\mathrm{s}}$, owing to enhanced electrostatic repulsion between neighboring charged groups of the chain..$^{2-5}$ If the polyelectrolyte is intrinsically flexible, this increase in backbone stiffness is attended by chain expansion due to electrostatic excluded-volume interactions between charged groups distant along the chain contour. Thus, experimental studies of excluded-volume and stiffness effects are essential for our understanding of polyelectrolyte chains in solution. In practice, however, there is a great difficulty that the two effects affecting the molecular size in a similar manner can hardly be separated by experiment without resort to a relevant excluded-volume theory.

In previous work, ${ }^{6,7}$ we estimated $q$ and $B$ (the excluded-volume strength) for sodium poly(2-acrylamido-2-methylpropanesulfonate) (Na PAMPS), an intrinsically flexible polyelectrolyte, in 0.05 and $0.5 \mathrm{M}$ aqueous $\mathrm{NaCl}$ from data for the intrinsic viscosity $[\eta]$, the mean-square radius of gyration, and the translational diffusion coefficient with the aid of the quasitwo-parameter (QTP) theory ${ }^{8-10}$ for the wormlike chain or, more generally, the helical wormlike chain. ${ }^{10}$ We found that this theoretical scheme for nonionic chains is capable of consistently describing excluded-volume effects on the three properties of the polymer in the aqueous salts. Thus the QTP theory should be useful for the study of dilute polyelectrolyte solu- tions unless $C_{\mathrm{s}}$ is too low.

The present work was undertaken to investigate the electrostatic contributions to chain stiffness and excluded-volume effects in aqueous $\mathrm{NaCl}$ solutions of $\mathrm{Na}$ PAMPS by viscometry. It is an extension of our previous studies on Na hyaluronte ${ }^{11-14}$ (a weakly stiff polysaccharide) and $\mathrm{Na}$ poly(styrenesulfonate), ${ }^{15-17}$ for which the ionic strength dependence of $q$ and $B$ was determined from measured $[\eta]$ with the aid of the QTP theory. In the work reported below, these parameters for Na PAMPS are estimated as functions of $C_{\mathrm{s}}$ in the range from 0.005 to $1 \mathrm{M}$ and compared with predictions from polyeletrolyte theories. ${ }^{2-5,18}$

\section{EXPERIMENTAL}

\section{Samples}

PAMPS samples ${ }^{6}$ previously synthesized and stored in the $\mathrm{Na}$ salt form were subjected to repeated fractional precipitation with $0.1 \mathrm{M}$ aqueous sodium acetate as the solvent and acetone as the precipitant in the manner described in ref 6 . From a number of fractions obtained, nine appropriate middle ones, designated below as F-H1, F-H2, .., F-H9 in the order of decreasing molecular weight, were chosen for the present work. These fractions were again converted to the Na salt form and dried as described previously. ${ }^{6}$ In addition to them, the previously investigated $\mathrm{Na}$ salt samples, ${ }^{6} \mathrm{f}-9-\mathrm{f}-14$, were used for viscosity measurements.

${ }^{\dagger}$ To whom correspondence should be addressed (E-mail: norisuye@chem.sci.osaka-u.ac.jp). 
Weight-average molecular weights $M_{\mathrm{w}}$ and z- to weight-average molecular weight ratios $\left(M_{\mathrm{z}} / M_{\mathrm{w}}\right)$ for samples F-H1, .., F-H9 were determined by sedimentation equilibrium with $0.5 \mathrm{M}$ aqueous $\mathrm{NaCl}$ at $25^{\circ} \mathrm{C}$ as the solvent. The experimental procedures were the same as those employed previously ${ }^{6}$ (see ref 19 for the determination of $M_{\mathrm{z}} / M_{\mathrm{w}}$ ).

\section{Viscometry}

Zero-shear rate intrinsic viscosities of Na PAMPS samples in $0.005-1 \mathrm{M}$ aqueous $\mathrm{NaCl}$ solutions at $25^{\circ} \mathrm{C}$ were determined using a low-shear four-bulb viscometer of the Ubbelohde type for the two highest molecular weight samples F-H1 at $C_{\mathrm{s}}=0.005$ and $0.01 \mathrm{M}$ and F-H2 at $C_{\mathrm{s}}=0.005 \mathrm{M}$ and conventional capillary viscometers of the Ubbelohde type for the rest. In evaluation of the relative viscosity $\eta_{\mathrm{r}}$, the difference between the solution and solvent densities was taken into account with the density increment $\partial \rho / \partial c$, which was obtained by interpolation or extrapolation of measured $\partial \rho / \partial c$ at $25^{\circ} \mathrm{C}(0.399,0.394$, and 0.370 at $C_{\mathrm{s}}=0.05,0.5$, and $1.5 \mathrm{M}$, respectively). Here, $\rho$ and $c$ denote the solution density and the polymer mass concentration, respectively.

The Huggins plot of $\eta_{\mathrm{sp}} / c v s . c$ was normal regardless of $C_{\mathrm{s}}$ for high molecular weights $\left(\eta_{\mathrm{sp}}\right.$ denotes the specific viscosity). As the molecular weight decreased, it became to have (1) much stronger $c$-dependence (with Huggins' constant larger than 0.7), (2) a maximum, or (3) a negative slope in the $c$ range $(0.06<$ $\left.\eta_{\mathrm{sp}}<0.6\right)$ of our experiment, showing typically observed polyelectrolyte behavior at low ionic strength (see Figure 1 of ref 16). In case (1), we were able to determine $[\eta]$ as far as the Huggins plot or the Mead-Fuoss plot of $\left(\ln \eta_{\mathrm{r}}\right) / c v s . c$ had a linear portion

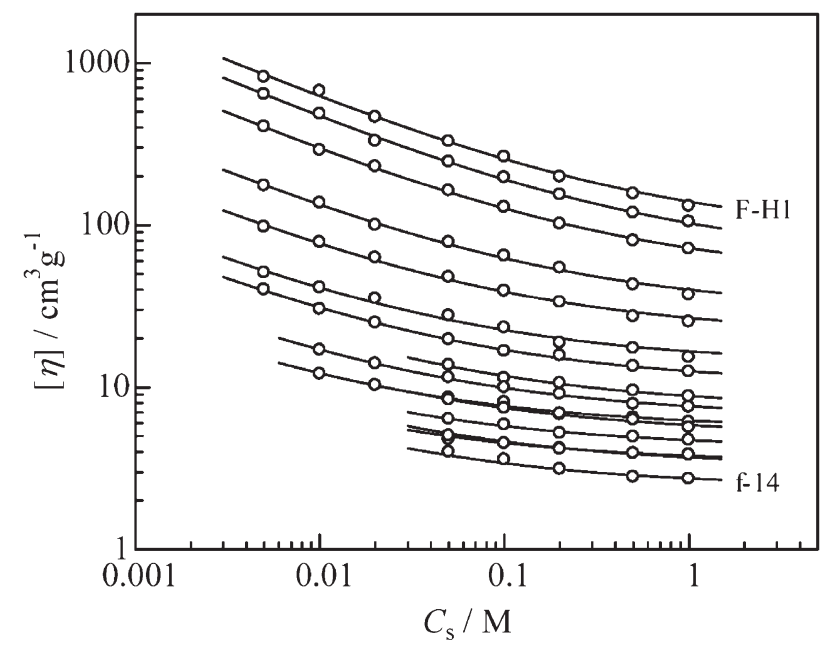

Figure 1. Dependence of $[\eta]$ on salt concentration for $\mathrm{Na}$ PAMPS samples (F-H1, F-H2, ..., F-H6, f-9, f-10, F-H7, f-11, F-H8, f-12, f-13, F-H9, and f-14 from top to bottom) in aqueous $\mathrm{NaCl}$ at $25^{\circ} \mathrm{C}$.
Table I. Results from sedimentation equilibrium measurements on Na PAMPS samples in $0.5 \mathrm{M}$ aqueous $\mathrm{NaCl}$ at $25^{\circ} \mathrm{C}$

\begin{tabular}{cccc}
\hline sample & $M_{\mathrm{w}} \times 10^{-4}$ & $A_{2} \times 10^{4} / \mathrm{mol} \mathrm{g}^{-2} \mathrm{~cm}^{3}$ & $M_{\mathrm{z}} / M_{\mathrm{w}}$ \\
\hline F-H1 & 71.1 & 2.70 & 1.1 \\
F-H2 & 49.5 & 3.13 & 1.1 \\
F-H3 & 31.5 & 3.72 & 1.1 \\
F-H4 & 13.8 & 4.74 & $1.1_{5}$ \\
F-H5 & 8.65 & 5.59 & $1.0_{5}$ \\
F-H6 & 4.18 & 6.94 & 1.3 \\
F-H7 & 1.35 & 11.2 & 1.2 \\
F-H8 & 0.878 & 15.2 & 1.2 \\
F-H9 & 0.287 & 31.9 & 1.2 \\
\hline
\end{tabular}

in a low $c$ region, but for $M_{\mathrm{w}}<5 \times 10^{3},[\eta]$ values obtained were less accurate since neither plot exhibited a definitely linear region even at the highest $C_{\mathrm{s}}$ studied. Cases (2) and (3) gave no reliable $[\eta]$ data, and as the result, we obtained [ $\eta$ ] only for $M_{\mathrm{w}}>3 \times$ $10^{4}$ at $C_{\mathrm{s}}=0.005 \mathrm{M}$ and for $M_{\mathrm{w}}>1 \times 10^{4}$ at $C_{\mathrm{s}}=$ 0.01 and $0.02 \mathrm{M}$.

\section{RESULTS AND DISCUSSION}

\section{Viscosity Behavior}

Table I summarizes results of $M_{\mathrm{w}}, A_{2}$ (the second virial coefficient), and $M_{\mathrm{z}} / M_{\mathrm{w}}$ from sedimentation equilibrium measurements. The molecular weight ratios indicate that our samples except F-H6 are fairly narrow in molecular weight distribution. The $A_{2}$ data (not graphically shown) fall on the previously established $\log A_{2}$ vs. $\log M_{\mathrm{w}}$ relation $^{6}$ for $0.5 \mathrm{M}$ aqueous $\mathrm{NaCl}$ of Na PAMPS covering a broad $M_{\mathrm{w}}$ range from $1.7 \times 10^{3}$ to $1.4 \times 10^{6}$.

Intrinsic viscosity data for all Na PAMPS samples in aqueous $\mathrm{NaCl}$ at $25^{\circ} \mathrm{C}$ are summarized in Table II, along with the previous ${ }^{6}$ and present $M_{\mathrm{w}}$ data, and are plotted double-logarithmically against $C_{\mathrm{s}}$ in Figure 1. With a decrease in $C_{\mathrm{s}}$, the curve fitting the data points for each $C_{\mathrm{s}}$ rises more pronouncedly for a higher molecular weight and in a lower $C_{\mathrm{s}}$ region. This behavior of $[\eta]$, due to enhanced electrostatic repulsion between charged groups of the polyelectrolyte chain, is similar to the previously observed $C_{\mathrm{s}}$-dependence for $\mathrm{Na}$ hyaluronate in aqueous $\mathrm{NaCl} .{ }^{12}$ Interestingly, however, it is partly contrasted to that for Na PSS, ${ }^{16}$ for which the increases in $[\eta]$ (with lowering $C_{\mathrm{s}}$ ) were more pronounced in the higher $C_{\mathrm{s}}$ region owing probably to a greater reduction in the attractive contribution to excluded-volume effects. Note that Na PSS attains the theta state in aqueous $\mathrm{NaCl}$ of $C_{\mathrm{s}}=4.17 \mathrm{M}$ at $16.4{ }^{\circ} \mathrm{C} .{ }^{15}$

Figure 2 illustrates the molecular weight dependence of $[\eta]$ at fixed $\mathrm{NaCl}$ concentrations. All the curves are essentially linear down to $M_{\mathrm{w}}=3 \times 10^{4}$, 
Table II. Intrinsic viscosity data for $\mathrm{Na}$ PAMPS samples in aqueous $\mathrm{NaCl}$ at $25^{\circ} \mathrm{C}$

\begin{tabular}{|c|c|c|c|c|c|c|c|c|c|}
\hline \multirow{3}{*}{ sample } & \multirow{3}{*}{$M_{\mathrm{w}} \times 10^{-4}$} & \multicolumn{8}{|c|}{$[\eta] / 10^{2} \mathrm{~cm}^{3} \mathrm{~g}^{-1}$} \\
\hline & & \multicolumn{8}{|c|}{$C_{\mathrm{s}} / \mathrm{M}$} \\
\hline & & 0.005 & 0.01 & 0.02 & 0.05 & 0.1 & 0.2 & 0.5 & 1 \\
\hline F-H1 & 71.1 & 8.17 & 6.70 & 4.64 & 3.28 & 2.64 & 1.99 & 1.56 & 1.31 \\
\hline F-H2 & 49.5 & 6.40 & 4.85 & 3.30 & 2.46 & 1.97 & 1.54 & 1.19 & 1.05 \\
\hline F-H3 & 31.5 & 4.05 & 2.90 & 2.30 & 1.63 & 1.29 & 1.02 & 0.801 & 0.716 \\
\hline F-H4 & 13.8 & 1.75 & 1.37 & 1.00 & 0.784 & 0.647 & 0.545 & 0.430 & 0.372 \\
\hline F-H5 & 8.65 & 0.976 & 0.786 & 0.630 & 0.479 & 0.393 & 0.335 & 0.273 & 0.253 \\
\hline F-H6 & 4.18 & 0.510 & 0.412 & 0.352 & 0.276 & 0.233 & 0.188 & 0.174 & 0.153 \\
\hline$f-9$ & $3.31^{\mathrm{a}}$ & 0.400 & 0.303 & 0.250 & $0.197^{\mathrm{a}}$ & 0.167 & 0.157 & $0.135^{\mathrm{a}}$ & 0.125 \\
\hline$f-10$ & $1.79^{\mathrm{a}}$ & - & - & - & $0.137^{\mathrm{a}}$ & 0.114 & 0.106 & $0.0955^{\mathrm{a}}$ & 0.0880 \\
\hline F-H7 & 1.35 & - & 0.170 & 0.140 & 0.115 & 0.100 & 0.0912 & 0.0788 & 0.0758 \\
\hline$f-11$ & $1.02^{\mathrm{a}}$ & - & 0.121 & 0.103 & $0.0865^{\mathrm{a}}$ & 0.0810 & 0.0680 & $0.0650^{\mathrm{a}}$ & 0.0610 \\
\hline F-H8 & 0.878 & - & - & - & 0.0840 & 0.0745 & 0.0690 & 0.0630 & 0.0565 \\
\hline$f-12$ & $0.711^{\mathrm{a}}$ & - & - & - & $0.0636^{\mathrm{a}}$ & 0.0587 & 0.0520 & $0.0495^{\mathrm{a}}$ & 0.0473 \\
\hline$f-13$ & $0.301^{\mathrm{a}}$ & - & - & - & $0.0503^{\mathrm{a}}$ & 0.0451 & 0.0416 & $0.0392^{\mathrm{a}}$ & 0.0383 \\
\hline F-H9 & 0.287 & - & - & - & 0.0478 & - & 0.042 & 0.0390 & 0.0385 \\
\hline$f-14$ & $0.172^{\mathrm{a}}$ & - & - & - & $0.0399^{a}$ & 0.0358 & 0.0313 & $0.0280^{\mathrm{a}}$ & 0.0273 \\
\hline
\end{tabular}

${ }^{\mathrm{a}}$ Taken from ref 6.

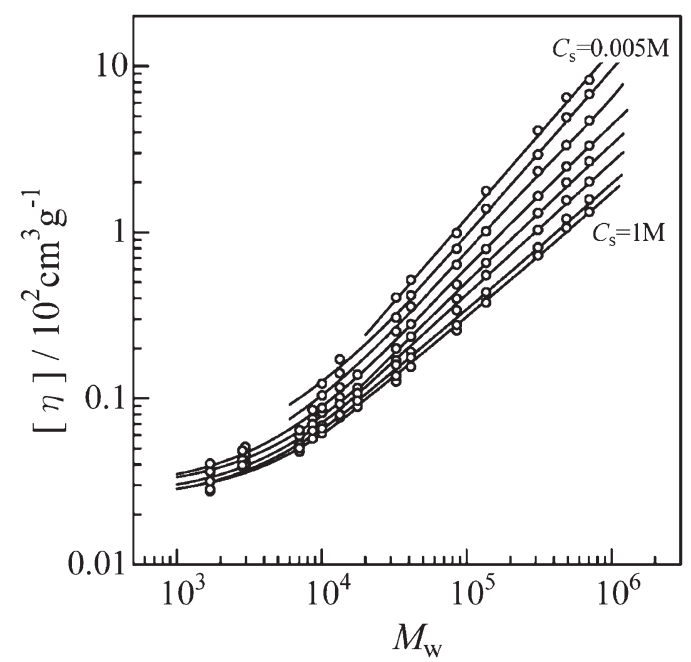

Figure 2. Molecular weight dependence of $[\eta]$ for Na PAMPS in aqueous $\mathrm{NaCl}\left(C_{\mathrm{s}}=0.005,0.01,0.02,0.05,0.1,0.2,0.5\right.$, and $1 \mathrm{M}$ from top to bottom) at $25^{\circ} \mathrm{C}$.

and those for $C_{\mathrm{s}} \geq 0.05 \mathrm{M}$ bend up with further decreasing $M_{\mathrm{w}}$. The viscosity exponent in the region of $M_{\mathrm{w}}$ above $3 \times 10^{4}$ increases from 0.77 (at $1 \mathrm{M}$ ) to 1.0 (at $0.005 \mathrm{M}$ ) with lowering $C_{\mathrm{s}}$ and is fairly close to what was early estimated by Fisher et al. ${ }^{20}$ in a narrow $M_{\mathrm{w}}$ range from $1.4 \times 10^{5}$ to $8.7 \times 10^{5}$. We note that our previous $[\eta]$ data at $C_{\mathrm{s}}=0.05$ and $0.5 \mathrm{M}$ (not shown) fall essentially on the corresponding curves in the figure.

\section{Data Analysis and Comparison with Theory}

The theory of Yoshizaki et al. ${ }^{21}$ for the intrinsic viscosity $[\eta]_{0}$ of an unperturbed wormlike touched-bead chain contains three parameters, $M_{\mathrm{L}}$ (the molar mass per unit contour length of the chain), $q$, and $d$ (the bead diameter). The first parameter is related to the molecular weight $M$ by $M_{\mathrm{L}}=M / L$, with $L$ being the contour length. The expansion factor $\alpha_{\eta}^{3}$ for $[\eta]$ in the QTP scheme may be expressed by

$$
\alpha_{\eta}^{3}=\left(1+3.8 \tilde{z}+1.9 \tilde{z}^{2}\right)^{0.3}
$$

if the Barrett equation ${ }^{22}$ is adopted. Here, $\tilde{z}$ is the scaled excluded-volume parameter defined by ${ }^{9,10}$

$$
\tilde{z}=(3 / 4) K\left(L_{r}\right) z
$$

with

$$
\begin{gathered}
K\left(L_{r}\right)=(4 / 3)-2.711\left(L_{r}\right)^{-1 / 2}+(7 / 6)\left(L_{r}\right)^{-1} \\
\text { for } L_{r}>6 \\
=\left(L_{r}\right)^{-1 / 2} \exp \left[-6.611\left(L_{r}\right)^{-1}\right. \\
\left.+0.9198+0.03516 L_{r}\right] \quad \text { for } L_{r} \leq 6 \\
\quad z=(3 / 2 \pi)^{3 / 2}(B / 2 q)\left(L_{r}\right)^{1 / 2}
\end{gathered}
$$

and

$$
L_{r}=L / 2 q
$$

The excluded-volume strength in eq 4 is defined (for the wormlike chain) by

$$
B=\beta / a^{2}
$$

where $\beta$ and $a$ are the binary cluster integral and the bead spacing, respectively. As may be seen from the above description, the four parameters, $q, M_{\mathrm{L}}, d$, and $B$, need to be determined for the evaluation of theoretical $[\eta]$ for a given $M$.

With the previous $M_{\mathrm{L}}$ value $^{6}$ of $900 \mathrm{~nm}^{-1}$ at $C_{\mathrm{s}}=0.05$ and $0.5 \mathrm{M}$ (see ref 6 for its estimation and 


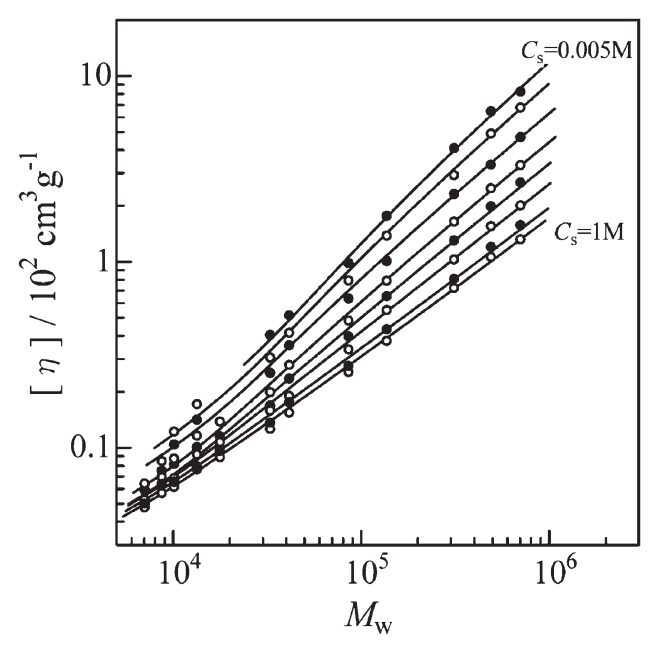

Figure 3. Comparison between measured $[\eta]$ for Na PAMPS in aqueous $\mathrm{NaCl}$ at $25^{\circ} \mathrm{C}$ and the theoretical curves computed from the theory of Yoshizaki et al. ${ }^{21}$ for unperturbed wormlike touched-bead chains and the QTP theory ${ }^{8-10}$ (eqs 1-6) for excluded-volume effects with the parameters in Table III.

Table III. Wormlike-chain parameters and excluded-volume strength for $\mathrm{Na}$ PAMPS in aqueous $\mathrm{NaCl}$ at $25^{\circ} \mathrm{C}$

\begin{tabular}{lcccc}
\hline$C_{\mathrm{s}} / \mathrm{M}$ & $M_{\mathrm{L}} / \mathrm{nm}^{-1}$ & $q / \mathrm{nm}$ & $B / \mathrm{nm}$ & $d / \mathrm{nm}$ \\
\hline 0.005 & $900^{\mathrm{a}}$ & 8.0 & 17 & 2.4 \\
0.01 & $900^{\mathrm{a}}$ & 6.0 & 13 & 2.2 \\
0.02 & $900^{\mathrm{a}}$ & 4.3 & 8.5 & 2.0 \\
0.05 & $900^{\mathrm{a}}$ & $3.0^{\mathrm{a}}$ & $6.2^{\mathrm{a}}$ & $1.7^{\mathrm{a}}$ \\
0.1 & $900^{\mathrm{a}}$ & 2.2 & 5.0 & 1.6 \\
0.2 & $900^{\mathrm{a}}$ & 1.9 & 3.5 & 1.6 \\
0.5 & $900^{\mathrm{a}}$ & $1.4^{\mathrm{a}}$ & $2.7^{\mathrm{a}}$ & $1.5_{5}^{\mathrm{a}}$ \\
1 & $900^{\mathrm{a}}$ & 1.3 & 2.3 & 1.5 \\
\hline
\end{tabular}

${ }^{\mathrm{a}}$ Taken from ref 6.

uncertainty), we searched for a set of $q, d$, and $B$ leading to the best fit to the $[\eta]$ data at each $C_{\mathrm{s}}$ (except 0.05 and $0.5 \mathrm{M}$ ) in Figure 2; the less accurate data for $M_{\mathrm{w}}<4 \times 10^{3}$ were not used for the fitting (see the remarks in the EXPERIMENTAL section). At $C_{\mathrm{s}} \leq$ $0.02 \mathrm{M}$, at which the $[\eta]$ data are limited to relatively high $M_{\mathrm{w}}, d$ was successively changed from the previously estimated value $1.7 \mathrm{~nm}$ at $C_{\mathrm{s}}=0.05 \mathrm{M}$ until the closest agreement was reached. The fitting results are shown in Figure 3, in which the theoretical curves have been computed with the parameters summarized in Table III. The agreement is fairly good in the indicated molecular weight range. The uncertainty of each parameter in the table is of the order of $\pm 10 \%$ or a little more for $d$ and at $0.005 \mathrm{M}$. The value of $d$ tends to increase with lowering $C_{\mathrm{s}}$, but the significance of this trend is unclear to us. We also note that the $M_{\mathrm{L}}$ of $900 \mathrm{~nm}^{-1}$ is close to the value $920 \mathrm{~nm}^{-1}$ expected for the polyelectrolyte chain in the all trans conformation and gives $h$ (the contour length per monomer residue) a value of $0.26 \mathrm{~nm}$.
Table III shows that both chain stiffness and excluded-volume strength increase with lowering ionic strength. This increase in $q$ may be taken as that in the electrostatic persistence $q_{\mathrm{el}}$, i.e., the electrostatic contribution to the backbone stiffness; according to the Odijk-Skolnick-Fixman (OSF) theory, ${ }^{2,3} q$ is equal to the sum of $q_{\mathrm{el}}$ and $q_{0}$ (the intrinsic persistence length). It is important to note, however, that, since the total electrostatic interaction energy on the chain diminishes with shortening $L$, the electrostatic contribution is a decreasing function of $\kappa L$ for short chains $^{2,23}$ ( $\kappa$ denotes the reciprocal Debye length defined for aqueous 1-1 salts by $\kappa^{2}=8 \pi Q_{\mathrm{B}} N_{\mathrm{A}} C_{\mathrm{s}} /$ 1000 , with $Q_{\mathrm{B}}$ and $N_{\mathrm{A}}$ being the Bjerrum length and the Avogadro constant, respectively). In other words, $q_{\text {el,app }}$ (the apparent value of $q_{\mathrm{el}}$ at finite $L$ ) and $q_{\text {app }}$ (the apparent value of $q$, i.e., $q_{\mathrm{app}}=q_{0}+q_{\mathrm{el}, \mathrm{app}}$ ) are equal to $q_{\mathrm{el}}$ and $q$, respectively, when $\kappa L$ is much larger than unity. In the previous work, ${ }^{6}$ we found that the estimation of $q$ is hardly affected by this end effect on $q_{\mathrm{el}}$ at $C_{\mathrm{s}}=0.05$ and $0.5 \mathrm{M}$, using $q_{0}=0.66 \mathrm{~nm}$ (a rough estimate) and the expression

$$
q_{\text {app }}=q_{0}+\left(q-q_{0}\right) P
$$

with $^{2,23}$

$$
\begin{aligned}
P \equiv & q_{\mathrm{el}, \mathrm{app}} / q_{\mathrm{el}}=1-(8 / 3)(\kappa L)^{-1} \\
& +(3 \kappa L)^{-1}\left(8+5 \kappa L+\kappa^{2} L^{2}\right) \exp (-\kappa L)
\end{aligned}
$$

Namely, the $[\eta]_{0}$ values lowered by decreases in (apparent) stiffness led to increases in excluded-volume effect, thereby bringing theoretical $[\eta]\left(=[\eta]_{0} \alpha_{\eta}^{3}\right)$ back nearly to the original values given by $q$ (the differences were at most a few \%).

The present analysis showed this to be also the case at any $C_{\mathrm{s}}$ higher than $0.05 \mathrm{M}$ (for $M>7 \times 10^{3}$ ) and at $C_{\mathrm{s}}=0.02$ and $0.01 \mathrm{M}$ (for $M>10^{4}$ ); use was made of the $q_{0}$ value of $0.66 \mathrm{~nm}$ determined by linear extrapolation of $q$ vs. $C_{\mathrm{s}}^{-1 / 2}$ to infinite ionic strength. At $C_{\mathrm{s}}=0.005 \mathrm{M}$, however, the lowered apparent stiffness surpassed the enhanced excluded-volume effect, resulting in lowering of theoretical $[\eta]$ by $3-6 \%$ from the original values for $M<8 \times 10^{4}$. Thus, the estimated parameters at this lowest $C_{\mathrm{s}}$ must be accepted with some reservation.

\section{Electrostatic Persistence Length}

The values of $q_{\mathrm{el}}$ for Na PAMPS calculated from the $q$ data in Table III with $q_{0}=0.66 \mathrm{~nm}$ are compared with the Le Bret theory ${ }^{4}$ based on the complete Poisson-Boltzmann equation and the OSF theory ${ }^{2,3}$ based on the Debye-Hückel (DH) approximation in Figure 4. In evaluating $q_{\mathrm{el}}$ from the former theory, we used values of $3.9 \mathrm{~nm}, 1.1 \mathrm{~nm}$, and 0 for the linear charge density $\sigma\left(=h^{-1}\right)$, the chain diameter $d_{0}$ (esti- 


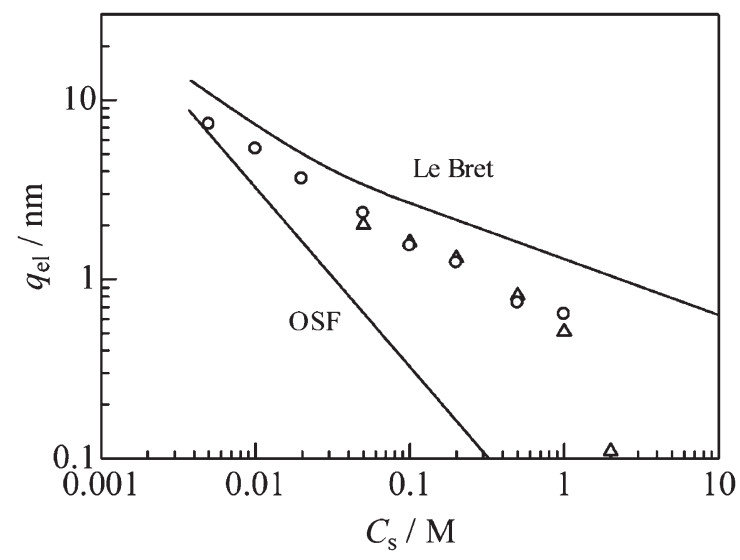

Figure 4. Comparison of experimental $q_{\mathrm{el}}$ for Na PAMPS (circles) in aqueous $\mathrm{NaCl}$ with the $\mathrm{Le} \mathrm{Bret}^{4}$ and $\mathrm{OSF}^{2,3}$ theories. The triangles represent previous $q_{\mathrm{el}}$ data (estimated from $[\eta]$ data) for $\mathrm{Na}$ PSS in aqueous $\mathrm{NaCl}^{16,17}$

mated from $\bar{v}=\pi N_{\mathrm{A}} d_{0}^{2} / 4 M_{\mathrm{L}}$ using the measured partial specific volumes $\bar{v}$ ), and the ratio of the dielectric constant of the polymer to that of water, respectively. In calculating OSF's $q_{\mathrm{el}}\left[=\left(4 \kappa^{2} Q_{\mathrm{B}}\right)^{-1}\right]$, we invoked the ion condensation hypothesis, ${ }^{24}$ since the charge parameter $\sigma Q_{\mathrm{B}}$ for Na PAMPS (=2.8) exceeds unity.

It can be seen in Figure 4 that the Le Bret theory overestimates $q_{\mathrm{el}}$ while the OSF theory underestimates it. The experimental $\log q_{\mathrm{el}}$ varies almost linearly with $\log C_{\mathrm{s}}$ with a slope of -0.48 , which is steeper than that $(-0.33)$ of the Le Bret curve for $C_{\mathrm{s}}>0.04 \mathrm{M}$ and much more gradual than that $(-1.00)$ of the OSF curve. The $q_{\mathrm{el}}$ data for Na PSS ${ }^{16,17}$ indicated by the triangles in the figure come close to those for $\mathrm{Na}$ PAMS in the $C_{\mathrm{s}}$ range between 0.05 and $1 \mathrm{M}$. This is because both $q$ and $q_{0}$ for the two polymers happen to be roughly the same at every $C_{\mathrm{s}}$ in that range $\left(q_{0}=0.66\right.$ and $0.69 \mathrm{~nm}$ for $\mathrm{Na}$ PAMPS and $\mathrm{Na}$ PSS, ${ }^{15,16}$ respectively). The two theoretical curves for Na PSS (not shown) are indistinguishable from the respective lines for Na PAMPS (note that the OSF curve is exactly the same for the two polymers). Thus, the extent of disagreement between theory and experiment for Na PAMPS is quite similar to what was early observed for Na PSS, confirming the previous conclusion ${ }^{16}$ that the Le Bret and OSF theories fail to describe the $\kappa$-dependence of $q_{\mathrm{el}}$ for intrinsically flexible polyelectrolytes.

\section{Excluded-Volume Strength}

The binary cluster integral appearing in eq 6 may be divided into three contributions from hard-core $\left(w_{\mathrm{c}}\right)$, electrostatic $\left(w_{\mathrm{el}}\right)$, and attractive $\left(w_{\mathrm{a}}\right)$ potentials acting on a pair of beads: ${ }^{18}$

$$
\beta=\beta_{\mathrm{c}}+\beta_{\mathrm{el}}+\beta_{\mathrm{a}}
$$

where

$$
\begin{aligned}
\beta_{\mathrm{c}}= & 4 \pi \int_{0}^{d_{\mathrm{c}}} r^{2} d r \\
\beta_{\mathrm{el}}= & 4 \pi \int_{d_{\mathrm{c}}}^{\infty} r^{2}\left[1-\exp \left(-w_{\mathrm{el}} / k_{\mathrm{B}} T\right)\right] d r \\
\beta_{\mathrm{a}}= & 4 \pi \int_{d_{\mathrm{c}}}^{\infty} r^{2} \exp \left(-w_{\mathrm{el}} / k_{\mathrm{B}} T\right) \\
& \times\left[1-\exp \left(-w_{\mathrm{a}} / k_{\mathrm{B}} T\right)\right] d r
\end{aligned}
$$

In these equations, $r$ is the bead-bead distance, $d_{\mathrm{c}}$ the hard-core diameter of each bead, $k_{\mathrm{B}}$ the Boltzmann constant, and $T$ the absolute temperature; the electrostatic and attractive contributions $\left(\beta_{\mathrm{el}}\right.$ and $\left.\beta_{\mathrm{a}}\right)$ have been separated from the function $\left\{1-\exp \left[-\left(w_{\mathrm{el}}+\right.\right.\right.$ $\left.\left.\left.w_{\mathrm{a}}\right) / k_{\mathrm{B}} T\right]\right\}$ for $r>d_{\mathrm{c}}$ where $w_{\mathrm{c}}=0$. The latter contribution expressed by eq 10c abruptly diminishes with decreasing ionic strength because of the presence of the term $\exp \left(-w_{\mathrm{el}} / k_{\mathrm{B}} T\right)$ and is often ignored in the evaluation of $\beta$ or $B$ for polyelectrolytes. ${ }^{12,18}$

We previously ${ }^{16}$ evaluated $B$ for Na PSS from eqs 6,9 , and 10 using the DH screened Coulomb potential $\left(Q_{\mathrm{B}} / r\right) \exp (-\kappa r)$ for $w_{\mathrm{el}} / k_{\mathrm{B}} T$ (with the ion condensation hypothesis ${ }^{24}$ ) and the familiar potential function - constant $/ r^{6}$ for $w_{\mathrm{a}} / k_{\mathrm{B}} T$, with the result that the numerically calculated $B$ is one order of magnitude larger than the experimental value at $C_{\mathrm{s}}=0.05$ $\mathrm{M}$ (the lowest $C_{\mathrm{s}}$ studied). The present work confirmed this to be also the case for Na PAMPS; the discrepancy amounted to a factor of 17 at $C_{\mathrm{s}}=0.005 \mathrm{M}$. These and similar earlier findings ${ }^{25,26}$ lend support to Fixman and Skolnick's idea ${ }^{18}$ that the conventional bead model is not directly applicable to the evaluation of $\beta_{\text {el }}$ for polyelectrolytes at low ionic strength where $\kappa^{-1}$ is comparable to or even larger than the bead spacing.

Fixman and Skolnick, ${ }^{18}$ replacing a set of beads by a rodlike segment with line charge distribution, evaluated the electrostatic contribution $\beta_{\mathrm{el}}^{\prime}$ to the excluded volume $\beta^{\prime}$ for a pair of segments of length $l$ and hardcore diameter $d_{\mathrm{c}}^{\prime}$ in the $\mathrm{DH}$ approximation (each prime symbol signifies the rodlike segment model). If $\beta^{\prime}$ for each segment consisting of $n_{0}$ beads $\left(l=n_{0} a\right)$ is equated to $n_{0}^{2} \beta,{ }^{18}$ it follows that $\beta / a^{2}=\beta^{\prime} / l^{2}$. The FixmanSkolnick (FS) theory ${ }^{18}$ may then be expressed in terms of $B$ as

$$
B=(\pi / 2) d_{\mathrm{c}}^{\prime}+(2 / \kappa) R(y)
$$

where the first and second terms represent the hardcore $\left(\beta_{\mathrm{c}}{ }^{\prime} / l^{2}\right)$ and electrostatic $\left(\beta_{\mathrm{el}}{ }^{\prime} / l^{2}\right)$ contributions, respectively (the attractive contribution $\beta_{\mathrm{a}}{ }^{\prime} / l^{2}$ to $B$ is ignored), and $R(y)$ is a known function of $y$, which is defined by $y=2 \pi\left(Q_{\mathrm{B}} \kappa\right)^{-1} \exp \left(-\kappa d_{\mathrm{c}}{ }^{\prime}\right)$ if ion condensation ${ }^{24}$ is considered. We note that the end effects, neglected in eq 11, may not be very important since both ends of each segment are linked to the ad- 


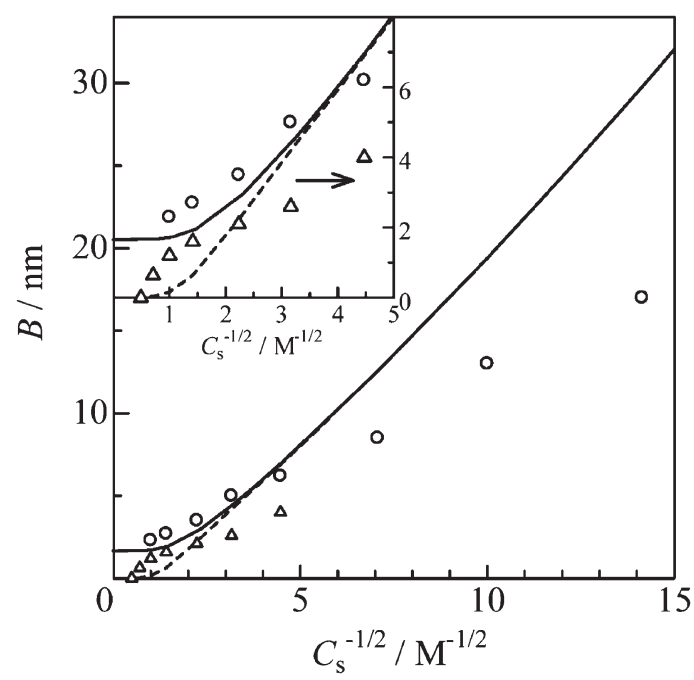

Figure 5. Comparison of experimental $B$ for Na PAMPS (circles) in aqueous $\mathrm{NaCl}$ with Fixman and Skolnick's theoretical solid curve (eq 11 with $d_{\mathrm{c}}{ }^{\prime}=1.1 \mathrm{~nm}$ ) for the rodlike segment model. ${ }^{18}$ The triangles and the dashed line represent previous $B$ data for Na PSS ${ }^{16,17}$ and theoretical values ${ }^{16,18}$ (eq 11 with eq 12), respectively. The inset enlarges the high $C_{\mathrm{S}}$ region with the $B$ values indicated on the right ordinate.

jacent segments.

Figure 5 compares the $B$ data (the circles) for $\mathrm{Na}$ PAMPS in aqueous $\mathrm{NaCl}$ with the theoretical solid curve computed from eq 11 with $d_{\mathrm{c}}{ }^{\prime}=1.1 \mathrm{~nm}$. It can be seen that the FS theory considerably overestimates $B$ for $C_{\mathrm{s}}$ below $0.02 \mathrm{M}\left(C_{\mathrm{s}}^{-1 / 2}>7 \mathrm{M}^{-1 / 2}\right)$. The second term of eq 11 is responsible for this discrepancy since it is predominant in the low $C_{\mathrm{s}}$ region. It should be pointed out that without consideration of ion condensation, the theoretical $B$ is as large as $240 \mathrm{~nm}$ at $C_{\mathrm{s}}=0.005 \mathrm{M}$.

Our previous comparison ${ }^{16}$ of $B$ for Na PSS in aqueous $\mathrm{NaCl}$ is also shown in Figure 5. Since this system attains the theta state at $C_{\mathrm{s}}=4.17 \mathrm{M}\left(16.4^{\circ} \mathrm{C}\right),{ }^{15}$ the theoretical $B$ (the dashed line) contains the attractive contribution, which may be given by

$$
\begin{aligned}
\beta_{\mathrm{a}}{ }^{\prime} / l^{2}= & \int_{0}^{\pi} \sin ^{2} \theta d \theta \int_{d_{\mathrm{c}^{\prime}}}^{\infty} \exp \left(-w_{\mathrm{el}}{ }^{\prime} / k_{\mathrm{B}} T\right) \\
& \times\left[1-\exp \left(-w_{\mathrm{a}}{ }^{\prime} / k_{\mathrm{B}} T\right)\right] d x
\end{aligned}
$$

with

$$
\begin{aligned}
w_{\mathrm{el}}{ }^{\prime} / k_{\mathrm{B}} T & =(y / \sin \theta) \exp \left[-\kappa\left(x-d_{\mathrm{c}}{ }^{\prime}\right)\right] \\
w_{\mathrm{a}}{ }^{\prime} / k_{\mathrm{B}} T & =-A^{\prime} /\left(x^{4} \sin \theta\right)
\end{aligned}
$$

In these equations, $x$ and $\theta$ denote the shortest distance between the two rod axes and the angle between them, respectively, and $A^{\prime}$ is a positive constant (determined in such a way that $B$ vanishes at the theta point); eq 14 has been derived with the $r^{-6}$ function (per unit contour length). ${ }^{27}$ The inset enlarges the high $C_{\mathrm{s}}$ region with the $B$ values indicated on the right ordinate.
The agreement between theory and experiment is poor for Na PSS, too. Comparison with the case for $\mathrm{Na}$ PAMPS reveals that, while the dashed and solid curves merge at $C_{\mathrm{s}}^{-1 / 2} \sim 4 \mathrm{M}^{-1 / 2}$ (as $C_{\mathrm{s}}^{-1}$ increases or the attractive contribution diminishes), the plotted points for the two polymers do not come close to each other even at such low $C_{\mathrm{s}}$. Thus, the FS theory or its modification fails to explain even the difference in $B$ between these polymers at low $C_{\mathrm{s}}$ where electrostatic interactions predominantly contribute to $B$.

At a given $\kappa$ or $C_{\mathrm{s}}$, no substantial difference between Na PAMPS and Na PSS can be found in any physical parameters affecting the value of $(\pi / 2) d_{\mathrm{c}}{ }^{\prime}+$ $(2 / \kappa) R(y)$ in eq 11. Furthermore, neither $\sigma$ nor $q$ for the two polymers significantly differs (at least, in the $C_{\mathrm{s}}$ range concerned). Hence, the attractive contribution $\left(\beta_{\mathrm{a}}{ }^{\prime} / l^{2}\right)$ must be primarily responsible for the difference between the circles and the triangles in Figure 5. The theoretical curves in the figure are indeed consistent with this for $C_{\mathrm{s}}^{-1 / 2}<2 \mathrm{M}^{-1 / 2}$, but the attractive contribution important for Na PSS in that high $C_{\mathrm{s}}$ region becomes negligible at $C_{\mathrm{s}}^{-1 / 2} \sim$ $3 \mathrm{M}^{-1 / 2}$. Since this contribution is too small at $C_{\mathrm{s}}^{-1 / 2}>3 \mathrm{M}^{-1 / 2}$ to be attributed to the poor approximation of $w_{\mathrm{el}}{ }^{\prime}$ (see eq 12), $w_{\mathrm{a}}{ }^{\prime}$ itself in eq 12 should be responsible for $\beta_{\mathrm{a}}{ }^{\prime} / l^{2}$. In such a low $C_{\mathrm{s}}$ region, negative $w_{\mathrm{a}}{ }^{\prime}$ must be an increasing function of $C_{\mathrm{s}}^{-1 / 2}$ rather than a constant independent of $C_{\mathrm{s}}$ (see eq 14) so that $\beta_{\mathrm{a}}{ }^{\prime} / l^{2}$ remains significant at $C_{\mathrm{s}}^{-1 / 2}>3 \mathrm{M}^{-1 / 2}$. If specific attractions (between hydrophobic portions of the Na PSS chain) are induced indirectly by electrostatic repulsion owing to some spatial constraint, negative $w_{\mathrm{a}}{ }^{\prime}$ may have such a $C_{\mathrm{s}}$-dependence. At present, we are unable to discuss more than this speculation. The observed difference in $B$ between Na PAMPS and Na PSS seems informative for our understanding of intramolecular excluded-volume effects in view of the close similarity of the two polymers in all conformational or structural characteristics except that associated with attractions.

\section{CONCLUSIONS}

The molecular weight dependence of $[\eta]$ for $\mathrm{Na}$ PAMPS in aqueous $\mathrm{NaCl}$ at fixed salt concentrations (between 0.005 and $1 \mathrm{M}$ ) is fairly satisfactory described by a combination of Yoshizaki et al.'s theory ${ }^{21}$ for unperturbed wormlike chains and the quasi-twoparameter theory ${ }^{8-10}$ for excluded-volume effects in nonionic chains. The persistence length and its electrostatic contribution $q_{\mathrm{el}}$ are similar to those for $\mathrm{Na}$ $\mathrm{PSS}^{16}$ in their magnitude and $C_{\mathrm{s}}$-dependence, while the excluded-volume strength $B$ considerably differs between the two polymers. As was the case for $\mathrm{Na}$ PSS, the available polyelectrolyte theories fail to de- 
scribe the $C_{\mathrm{s}}$-dependence of $q_{\mathrm{el}}$ and $B$ for Na PAMPS. Those for $B$ do not explain even the difference in $B$ between the two polyelectrolytes at low $C_{\mathrm{s}}$.

\section{REFERENCES}

1. O. Kratky and G. Porod, Recl. Trav. Chim. Pays-Bas, 68, 1106 (1949).

2. T. Odijk, J. Polym. Sci., Polym. Phys. Ed., 15, 477 (1977).

3. J. Skolnick and M. Fixman, Macromolecules, 10, 944 (1977).

4. M. Le Bret, J. Chem. Phys., 76, 6243 (1982).

5. M. Fixman, J. Chem. Phys., 76, 6346 (1982).

6. J. Yashiro, R. Hagino, S. Sato, and T. Norisuye, Polym. J., 38, 57 (2006).

7. J. Yashiro and T. Norisuye, Polym. Bull., 56, 467 (2006).

8. H. Yamakawa and W. H. Stockmayer, J. Chem. Phys., 57, 2843 (1972).

9. J. Shimada and H. Yamakawa, J. Chem. Phys., 85, 591 (1986).

10. H. Yamakawa, "Helical Wormlike Chains in Polymer Solutions," Springer, Berlin, 1997.

11. K. Hayashi, K. Tsutsumi, F. Nakajima, T. Norisuye, and A. Teramoto, Macromolecules, 28, 3824 (1995).

12. K. Hayashi, K. Tsutsumi, T. Norisuye, and A. Teramoto, Polym. J., 28, 922 (1996).
13. K. Tsutsumi and T. Norisuye, Polym. J., 30, 345 (1998).

14. N. Mizukoshi and T. Norisuye, Polym. Bull., 40, 555 (1998).

15. E. Hirose, Y. Iwamoto, and T. Norisuye, Macromolecules, 32, 8629 (1999).

16. Y. Iwamoto, E. Hirose, and T. Norisuye, Polym. J., 32, 428 (2000).

17. J. Yashiro and T. Norisuye, J. Polym. Sci., Part B: Polym. Phys., 40, 2728 (2002).

18. M. Fixman and J. Skolnick, Macromolecules, 11, 863 (1978).

19. T. Norisuye, T. Yanaki, and H. Fujita, J. Polym. Sci., Polym. Phys. Ed., 18, 547 (1980).

20. L. W. Fisher, A. R. Sochor, and J. S. Tan, Macromolecules, 10, 949 (1977).

21. T. Yoshizaki, I. Nitta, and H. Yamakawa, Macromolecules, 21, 165 (1988).

22. A. J. Barrett, Macromolecules, 17, 1566 (1984).

23. P. J. Hagerman, Biopolymers, 22, 811 (1983).

24. G. S. Manning, J. Chem. Phys., 51, 924 (1969).

25. I. Noda, T. Tsuge, and M. Nagasawa, J. Phys. Chem., 74, 710 (1970).

26. M. Nagasawa and A. Takahashi, in "Light Scattering from Polymer Solutions,” M. B. Huglin, Ed., Academic Press, New York, N. Y., 1972, Chapter 16.

27. K. Kawakami and T. Norisuye, Macromolecules, 24, 4898 (1991). 